\title{
Transcriptome analysis of Arabidopsis to predict stress length-specific regulatory networks involved in drought response
}

\author{
Sedigheh Akhtartavan ${ }^{1, *}$, Majid Talebi $^{2}$ \\ ${ }^{1}$ Department of Agricultural Biotechnology, Payame Noor University, Tehran, Iran \\ ${ }^{2}$ Department of Biotechnology, College of Agriculture, Isfahan University of Technology, Isfahan, Iran
}

*Corresponding author: akhtartava_s@yahoo.com

\begin{abstract}
Drought stress is one of the major environmental factors impairing crops productivity worldwide. Plants use various regulatory genes to reprogram genome activities to cope with such stresses. Among regulatory genes, transcription factors (TFs) function as terminal transducers and directly regulate the expression of wide spectrum of downstream genes. Multiplicity of the TF families and the complex interactions between TFs and cis-elements on the promoters of target genes as well as cross-talk between TFs in response to stress indicate the complexity of signaling networks involved in plant stress responses. This study aimed to use computational and statistical approaches to analyze a microarray dataset from Arabidopsis which covering different time periods of drought stress. After identifying and functional grouping of differentially expressed gens (DEGs), genes encoded TFs were determined and networked based on gene set enrichment analysis (GSE). Hierarchical regulatory network in each condition was assigned. After that, networks were used to conduct network topology analysis. Results indicated an obvious orientation in genome activity toward response to different cues; energy homeostasis and photosynthesis stability was occurred under drought stress. Also, 3787, 2931 and 5115 genes were differentially expressed under the early, moderate and prolonged drought stress, respectively, among them, 169, 140 and 261 TF were identified. Analysis of constructed regulatory networks of each drought condition revealed that plant recruits different but somewhat overlapping strategies to cope with stress in a long period of time. In each drought period, specific or common signaling pathways are activated using several numbers of transcription factors. It seems that among all identified TFs, ARR5, ARR6, ABF3, MYB29, MYB76 and SIGs genes are good candidate to manipulate plant stress tolerance.
\end{abstract}

Keywords: Regulatory networks, transcriptomics, drought stress, singular enrichment.

Abbreviations: TFs_Transcription factors; DEGs_Differentially expressed genes; GSE_gene set enrichment; ARR_Type-A Arabidopsis Response Regulators; $A B F 3 \_$ABF3 abscisic acid responsive elements-binding factor 3; MYB_myeloblastosis; SIG_Sigma factor; AP2/EREBP_APETALA2/ ethylene-responsive element binding proteins; NAC_NAM, ATAF, and CUC; b-ZIP_ Basic Leucine Zipper Domain; Dof_DNA binding with One Finger; bHLH_basic helix loop helix; HD-Zip_ Homeodomain-leucine zipper; HSF_Heat shock factor.

\section{Introduction}

Drought is a major factor impairing crops productivity worldwide (Farooq et al. 2012; Farooq et al. 2009). To cope with drought stress, plants use complex and multilayers mechanisms to reprogram their genome activity which results in change in genes expression and function (Ahuja et al. 2010; Ashraf 2010; Chen et al. 2014). Mainly, core regulatory networks underlying of such changes consist of several regulatory genes including transcription factors (TFs), protein kinases, protein phosphatases and miRNAs shaping the relevant transcriptome to show an adaptive response (Maruyama et al. 2012; Mehrotra et al. 2014; Mishra et al. 2006; Nakashima et al. 2009). Among all the mentioned components, TFs as one of the most important regulators, function as terminal transducers and directly regulate the expression of wide spectrum of downstream genes via binding to specific cis-elements in their promoter region (Yamaguchi-Shinozaki and Shinozaki 2005; Yamaguchi-
Shinozaki and Shinozaki 2006). In the recent decades, TFs gain the attention in researches that has been conducted to identify main tolerant genes (Gehan et al. 2015; Golldack et al. 2011; Maruyama et al. 2012). Several TF families including AP2/EREBP, MYB, WRKY, NAC, bZIP, Dof, bHLH, HD-Zip, HSF, etc, were identified and their roles in plant stress tolerance have been investigated (Gehan et al. 2015; Golldack et al. 2011; Yamaguchi-Shinozaki and Shinozaki 2006). However, it is not clear what $T F(s)$ in such a huge gene families should be selected to manipulate stress tolerant. This issue rises from the fact that stress response in plants is an extremely complicated process (Pessarakli 2011). Multiplicity of the gene families and the complex interactions between TFs and cis-elements on the promoters of target genes as well as cross-talk between TFs in response to stress indicate the complexity of signaling networks involved in plant stress responses (Seki et al. 2003; Shamloo- 
Dashtpagerdi et al. 2015; Tuberosa and Salvi 2006). Therefore, assigning the relations among TFs in response to drought stress will uncover the actual function of them facilitating improvement of drought tolerant crops. Microarray analysis is known as a powerful tool to discover and characterize genes involved in different stress responses in several plant species (Gul et al. 2016). Using this technology, it is possible to study of genome activity and identify regulation pattern of differentially-expressed TFs which may help us to study different pathways in drought stress condition. Although microarray analysis was used frequently to study plant response to drought stresses (Kathiresan et al. 2006; Mohanty et al. 2016; Rasheed et al. 2016) little is known about drought length specific regulatory networks. Characterization of TFs which function through a long drought period may introduce more reliable candidate gene(s) to use in crop stress tolerance manipulation. In this study, using computational approaches, a microarray dataset from Arabidopsis which covering different time periods of drought stress was analyzed. After assigning of differentially expressed genes (DEGs) followed by normalization and statistical analysis, ontology annotation was performed based on singular enrichment analysis. Furthermore, for each period, DEGs were determined. Several TFs were found among DEGs and their regulatory networks in each period were depicted. Results indicated that during drought stress, genome activity directed to response to internal and external cues and energy homeostasis, through regulatory complex and stress period specific regulatory networks are shaped during drought stress that had common and distinct TFs.

\section{Results}

\section{Statistical analysis of microarray data to find differentially} expressed genes

RMA algorithm normalized raw data successfully as the intensity value fitted well (Fig 1). ANOVA revealed that expression 6857 genes significantly changed during drought stress (p-value $\leq 0.05) \quad$ (Suppl Table 1). Paired wise comparison demonstrated that in contrast to normal condition, expression of 3787, 2931 and 5115 genes significantly differed in D1, D2 and D3 treatments, respectively ( $p$-value $\leq 0.05)$ that showed different magnitudes of expression changes (Suppl Table 2-4).

\section{Ontology analysis of DEGs}

Singular enrichment analysis was conducted for all DEGs identified by ANOVA to illustrate how the genome activities oriented in response to drought stress. From biological process aspect, DEGs were enriched in nine ontology groups (Fig 2-a). Results showed that during drought stress, genome activity was directed to metabolic process, translation and response to various internal and external cues such as "salt stress", "ABA", "cadmium ion", "cold" and "water deprivation" as well as photosynthesis. From point of view of molecular function ontology, a substantial of genome activities was related to regulatory and energy homeostasis mechanisms such as "protein binding", "transcription factor activity", "ATP binding", "catalytic activities", "Kinase activities" and so on (Fig 2-b), indicating that plant recruited genome activity reprograming as well as energy saving strategies to minimize adverse effects of drought stress.

\section{Identification of differentially expressed TFs}

From 3787 DEGs in D1, 169 genes were grouped in 42 distinct TF families (Table 1). NAC, AP2-EREB AND MYB families with 17, 14 and 14 members, respectively, comprised the largest groups. By contrast, 11 TF families contained only one gene (Suppl Table 5). 140 TFs belonged to 44 families were also identified among D2 DEGs (Table 1). Like as D1, NAC family had the most members (13 genes), while mTERF and AP2-EREB with 12 and 10 genes, respectively, were lied in next orders. Twenty one TF families had only one member in D2 (Suppl Tbale 6). A remarkable numbers of TFs was observed in D3 where, of 5115 DEGs, 261 TFs from 56 distinct families were assigned (Table 1). Unlike D1 and D2 conditions in which NAC family contained maximum number of genes, in D3, MYB family with 25 members had the most number of genes. NAC and AP2-EREB families with 19 and 18 members, respectively, were ranked as next families. Also, 13 families contained only one member in D3 (Suppl Table 7). Figure 3 represents the distribution of TF families among different drought periods. There was 35 common TF families among D1, D2 and D3. Furthermore, two families namely CSD and FHA were only observed in D1 (Fig 2). EIL, IWS1 and S1Fa-like families were only found in D2 (Fig 3). Nine TF families including ARID, BSD, Co-activator p15, CPP, DBP, FAR1, GeBP, PBF-2-Like and SRS specially existed in D3 (Fig 3).

\section{Analysis of regulatory networks}

Identified TFs in each drought period were networked and analyzed separately. So, three regulatory networks were obtained containing different number of nods. In all obtained networks, higher betweenness centrality is shown with bigger nodes and the closeness centrality is illustrated using gradient coloring in which, red and green color represent highest and lowest closeness centrality, respectively. The magnitude of edge betweenness centrality also is represented with higher edges thickness. Betweenness obtain by counting the number of shortest paths going through a specific nod which quantify the importance of node in a network (Boccaletti et al. 2006). The closeness of a node defined as the inverse of the average distance from all other nodes (Koschützki and Schreiber 2008). Also, edge betweenness considered as a standard measure of the impact of a node or a linkage in network and indicates to number of shortest paths between pairs of nodes that run through specific edge (Boccaletti et al. 2006; Koschützki and Schreiber 2008). Differentially expressed TFs between normal condition and D1 were located in a network with 52 nods and 67 edges (Fig 4-a). Topology analysis of network highlighted $A B A$, methyl jasmonate and $\mathrm{H}_{2} \mathrm{O}_{2}$ pathways among different signaling pathways indicating their important roles in early drought responses. In addition, Abscisic acid responsive elements-Binding Factor 3 (ABF3) and Type-A response regulator 6 (ARR6) transcription factors had the most centrality indices, respectively, implying their pivotal roles in predicted regulatory network. Expression of ABF3 significantly increased during all drought stress time points while ARR6 exhibited a decreased expression compared to normal condition (Suppl Table 1). 
Table 1.A summary of pairwise expression profiling and transcription factor found using Arabidopsis microarray data in drought stress. N, D1, D2 and D3 represent non stress, early drought, moderate drought and prolonged drought respectively. DEGs: Differentially expressed genes.

\begin{tabular}{llllllllll}
\hline & D1 & \multicolumn{1}{c}{ D2 } & \multicolumn{3}{c}{ D3 } \\
\cline { 2 - 9 } & DEGs & TFs & $\begin{array}{l}\text { TF } \\
\text { families }\end{array}$ & DEGs & TFs & $\begin{array}{l}\text { TF } \\
\text { families }\end{array}$ & DEGs & TFs & $\begin{array}{l}\text { TF } \\
\text { families }\end{array}$ \\
\hline $\mathrm{N}$ & 3787 & 169 & 42 & 2931 & 140 & 44 & 5115 & 261 & 56 \\
\hline
\end{tabular}

A

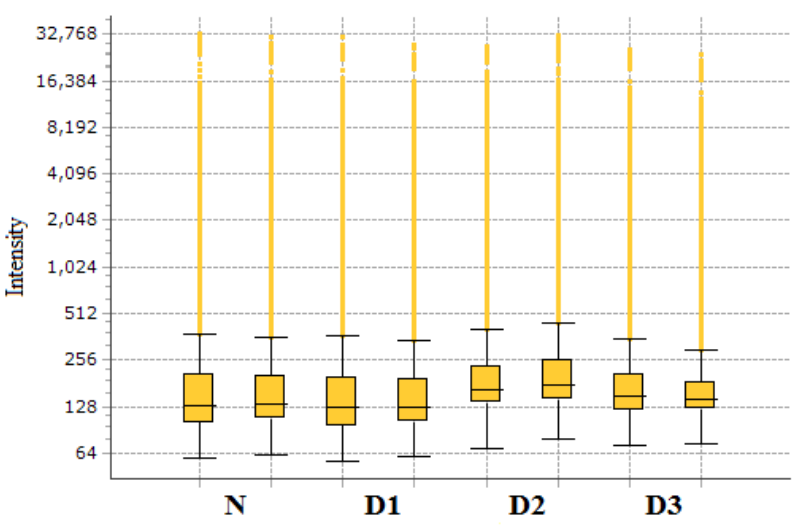

B

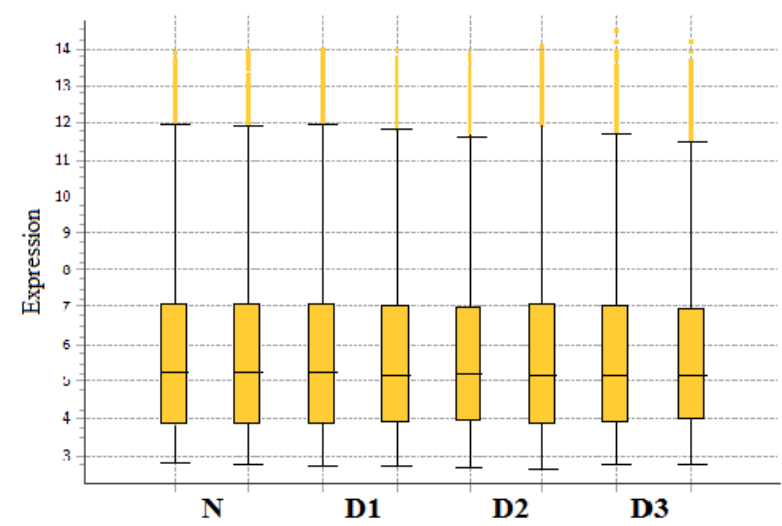

Fig 1. Normalization of microarray data of drought stress in Arabidopsis. A: Intensity values before normalization. B: Expression values after normalization. N, D1, D2 and D3 represent non stress, early drought, moderate drought and prolonged drought respectively.

A

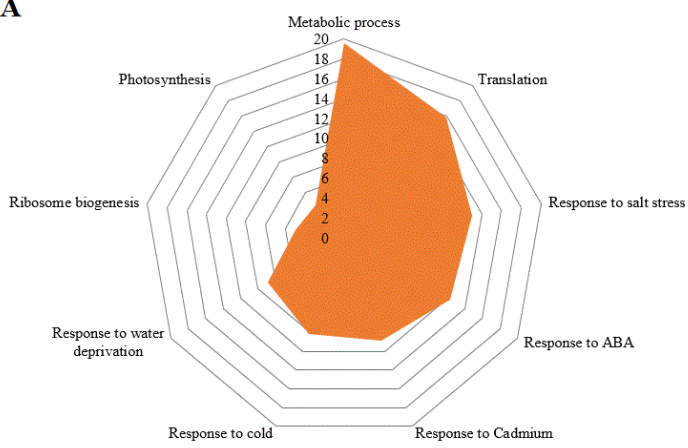

B

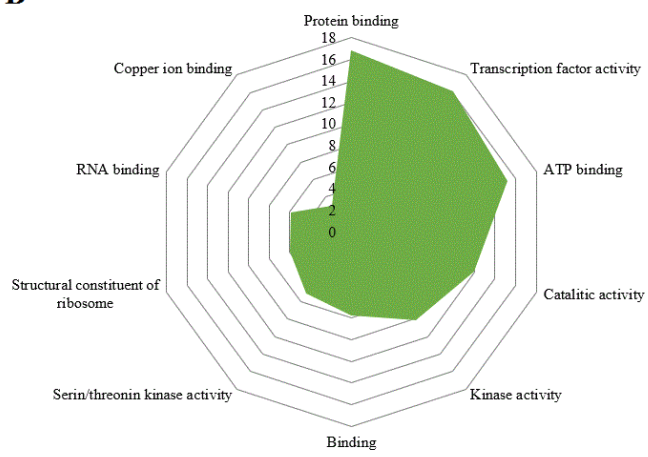

Fig 2. The percentages of functional modules of DEGs according to: A. biological process and B: molecular functions. 
Figure 3

$=\mathrm{D} 1 \quad=\mathrm{D} 2 \quad=\mathrm{D} 3$

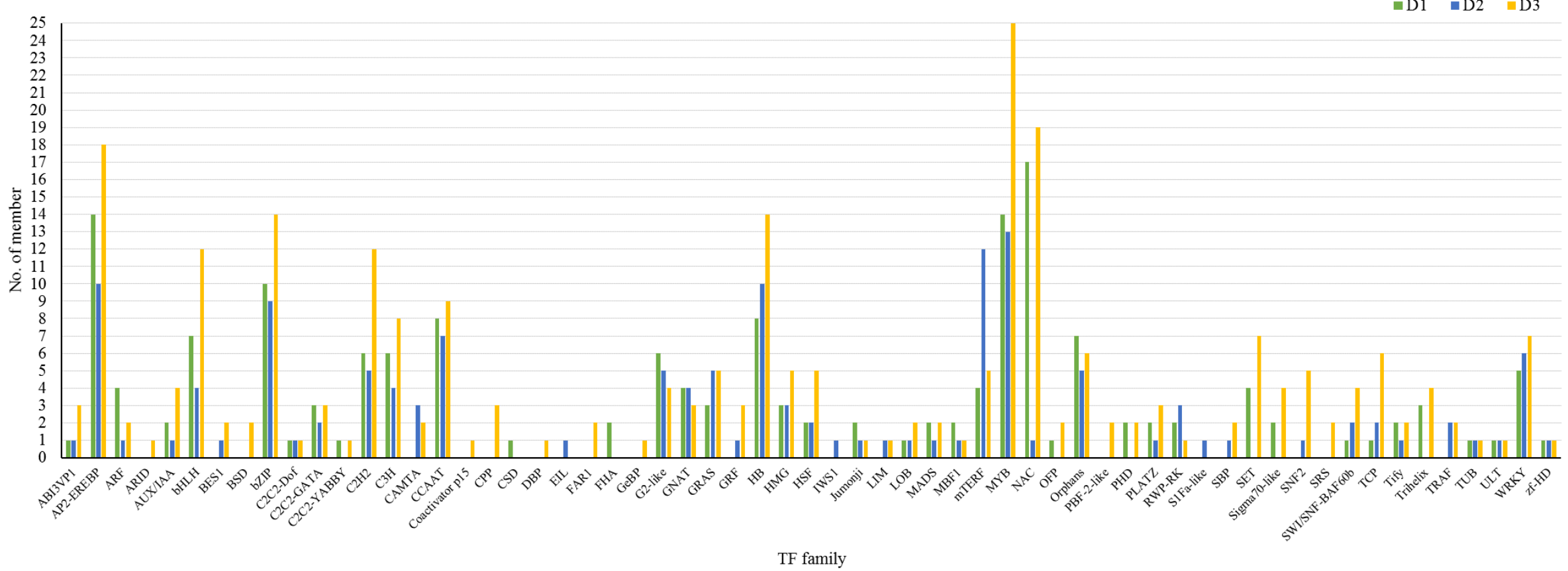

Fig. 3. Distribution of transcription families in three drought conditions. D1, D2 and D3 represent early drought, moderate drought and prolonged drought, respectively. 


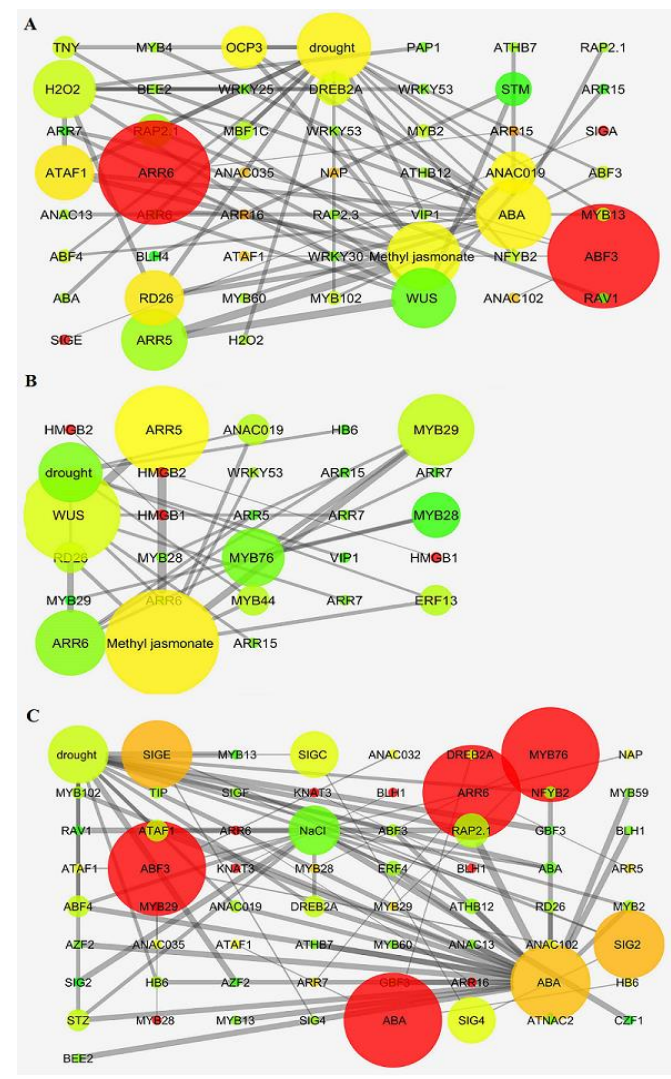

Fig 4. Topology analysis of predicted networks based on differentially expressed transcription factors. A: Network of early drought (D1). B: Network of moderate drought (D2) and C: Network of prolonged drought. In all three networks, nodes with bigger size indicate higher betweenness centrality. Red color represents highest and green color indicates the lowest closeness centrality. Edges with higher thickness reveal the high value of edge betweenness centrality.

Predicted regulatory network in D2 was consisted of 28 nods and 28 edges (Fig 4-b). In regulatory network of D2, only methyl jasmonate signaling pathway was bolded (Fig 4-b), considering the regulatory network in D1, this emphasizes significant impact of methyl jasmonate in response to average drought periods. Although ARR6 had a notable centrality indices, however, ARR5 and MYB29 genes were borne more centrality values in average in D2 network. A network with 65 nods and 74 edges was constructed in longterm drought stress (Fig 4-c). Interestingly, like as D1, ABA signaling was activated again in response to drought stress. Also, salinity stress signaling as $\mathrm{NaCl}$ term was highlighted in network which suggests that in response to prolonged drought condition, high osmotic and ionic stress signaling pathways are also activated. As shown in figure 3-c, three TFs namely $A B F 3, A R R 6$ and MYB76 gained most centrality values. Moreover, four members of plastid sigma factors (SIG) including SIG2, SIGC (SIG3), SIG4, and SIGE (SIG5) with relatively high centrality values were highlighted only in D3 networks. Statistical analysis of genes expression showed that all identified SIGs downregulated under drought stress.

\section{Discussion}

\section{Centralization of genome activity under drought stress}

Results of singular enrichment analysis obviously demonstrated that under drought stress, plant genome reprogramed to regulate mechanisms that enable plant to sense stress, transduce its signals and make changes in own physiology and biochemistry properties. The roles of TFs and protein kinases, as two major regulatory components (Duque et al. 2013), were emphasized along with the energy homeostasis and photosynthesis. The results were consistent with the fact that plant stress responses cause reduction in cellular energy and photosynthesis thereby plants need to constantly adjust their energy and photosynthesis-associated functions (Alqurashi et al. 2016; Yamori 2016). More details about undying molecular mechanisms of such alterations in genome activity are discussed below.

\section{Different signaling pathways in different drought periods}

In early drought treatment (D1), signaling pathways of $A B A$, methyl jasmonate and $\mathrm{H}_{2} \mathrm{O}_{2}$ were highlighted. The $A B A$ signaling pathway was also bolded in prolonged drought condition. Previous studies revealed that up to $25 \%$ of the Arabidopsis genes are affected by ABA and over $60 \%$ of the ABA-regulated genes are responsive to various abiotic stresses (Fujita et al. 2011; Zeller et al. 2009). Our results showed that NAC, AP2-EREB and MYB TF families were more represented among DEGs. Most of the members of these TF families are under the control of ABA (Golldack et al. 2011). $A B F 3$ which highlighted as main regulatory network gene in D1 and D3 conditions, is a b-ZIP protein that bind to the Gbox-type ABA Responsive Element (ABRE). ABF3 known as one of the core $A B A$ signaling components which is highly 
inducible by high salinity and osmotic stresses (Yoshida et al. 2010). Overexpression of Arabidopsis $A B F 3$ in transgenic rice increased tolerance to abiotic stresses (Oh et al. 2005). It has been revealed that one of the most important positive regulator of ABA signaling pathway namely SnRK2D/SnRK2.2 protein kinase interact with $A B F 3$ to active target genes in ABA signaling (Yoshida et al. 2010). From our results, it seems that during early drought condition, accumulation of $A B A$ and thereby activation of stress avoidance mechanisms such as stomatal closure, synthesis of osmolytes and etc. via $A B A$ responsive gens manily such as $A B F 3$, shape the dominant strategy to cope with drought. Highlighted $\mathrm{H}_{2} \mathrm{O}_{2}$ component reinforced our suggestion, because ROS act as intermediates in many ABA-regulated events at low concentrations and they act as signal molecules that triggers tolerance to stresses (Acharya and Assmann 2009; Quan et al. 2008). $\mathrm{H}_{2} \mathrm{O}_{2}$ can be induced by $A B A$ and accumulates in guard cells (Zhang et al. 2001) It has been shown that MAP kinases MPK3 and MPK6 induced by $\mathrm{H}_{2} \mathrm{O}_{2}$ (Jammes et al. 2009; Xing et al. 2008). Under osmotic stress MPK6 leads to stomatal closure and positive regulation of Heat shock factors (HSFs) (Liu 2012; Pérez-Salamó et al. 2014).

We found some evidences indicating that there is a crosstalk between Cytokinin (CK) and ABA signaling pathways in response to drought stress. Tow numbers of type-A ARR including ARR5 and ARR6 which known as main CK signaling components (Kunikowska et al. 2013), were delineated as one of the important networks components. Both genes exhibited decreased expression in drought stress. In a study, it has been demonstrated that individual arr5 and arr6 mutants in Arabidopsis had enhanced cold stress tolerance ( $\mathrm{Ha}$ et al. 2012). Moreover, expression of $A B 15$, an important $A B A$ signaling gene (Raghavendra et al. 2010), was negatively controlled by ARR4, ARR5 and ARR6 (Wang et al. 2011). So, these author suggested that these four proteins may form a protein complex to control the interplay between $C K$ and $A B A$ signaling in response to stresses (Wang et al. 2011). Altogether, it can be concluded that during drought stress, CK signaling suppressed and consequently, $A B A$ signaling intensified in overall through down-regulation of $A R R 6$ and ARR5 genes, triggering abiotic stress responses.

Topology analysis illustrated that besides the ABA and CK signaling pathways, methyl jasmonate pathway play important roles in plant adaptation to adverse conditions, particularly in D1 and D2 drought. Some previous reports suggested an involvement of methyl jasmonate with stress responses. For example, methyl jasmonate was reported to be involved in the adaptation of barley to salt stress (Walia et al. 2007). Also, it was associated with cold stress responses in the Thellungiella halophyla, a tolerant model plant (Wong et al. 2005). It seems that methyl jasmonate and ABA work synergistically or antagonistically in response to biotic and abiotic stresses. Under drought stress, methyl jasmonate modulates membrane lipid peroxidation and antioxidant activities to improve stress tolerance (Anjum et al. 2011). Furthermore, it has been demonstrated that $\mathrm{H}_{2} \mathrm{O}_{2}$ acts as secondary messenger in methyl jasmonate-induced genes transcription (Orozco-Cárdenas et al. 2001), which is in accordance with the our findings that indicated $\mathrm{H}_{2} \mathrm{O}_{2}$ and methyl jasmonate are the significance components of drought regulatory networks of D1 and D2 conditions. Interestingly, two significant identified MYB transcription factors namely MYB29 and MYB76 in D2 and D3 conditions, are involved in methyl jasmonate-mediated genes regulation (Gigolashvili et al. 2008). Soybean MYB76 conferred abiotic stresses tolerance in transgenic Arabidopsis through regulation of several stress-responsive genes (Liao et al. 2008). This gene was highlighted in both D2 and D3 conditions implying that it contributes in response to drought stress in both average and intense stress.

Exclusive highlighting of four members of plastid sigma factors namely SIG2, SIG3, SIG4 and SIG5 in D3 suggests a stress length specific fiction of these gens. All of the sigma factors from higher plants are nuclear encoded and regulate plastid gene transcription (Chi et al. 2015). Multiple sigma factors may have specialized or overlapping redundant functions based on the promoters they recognize (Chi et al. 2015). Based on expression and network topology analysis, prolonged drought stress may result in hampered plastid transcription which in turn, decreases plant photosynthetic capacity. These results are in accordance with results of singular enrichment analysis in which, photosynthesis associated genes were over-represented to compensate stress damages. Reduction in photosynthesis and consequently diminished final plant productivity due to abiotic stress, specially drought and heat stresses, are well documented (Xu et al. 2015). So, SIGs genes are one of the good candidates to manipulate photosynthetic pathway toward drought tolerance improvement.

\section{Materials and methods}

Plant materials such as species cultivar etc. that the data retrieved form databases. I also need authors to add at what stress condition of plant the data was generated (such as drought condition).

\section{Source of microarray data sets}

Raw microarray data of drought stress experiment (AC: EGEOD-24177) was downloaded from EBI (http://www.ebi.ac.uk/arrayexpress/). In brief, a controlled and sub-lethal moderate drought treatment system was used in Arabidopsis thaliana ecotype Columbia (Harb et al. 2010). Three drought treatment including 1 and 10 days after moderate drought as well as prolonged drought were considered (Harb et al. 2010).

\section{Expression profiling and ontology analysis}

Expression data was normalized using robust multi-array averaging algorithm (RMA) (Irizarry et al. 2003) via Expression Console 1.2 software (Affymetrix). Normal data was used in FlexArray 1.6 software (Blazejczyk et al. 2007). Analysis of variance (ANOVA) procedure was applied using same software to find DEGs through drought experiment. Furthermore, Bayesian t-test (Fox and Dimmic 2006) was used to paired wise comparison between experimental conditions (P-value $\leq 0.05)$. Gene ontology annotation was performed using GeneCodis (http://genecodis. cnb.csic.es/) (Tabas-Madrid et al. 2012), based on singular enrichment analysis (Nogales-Cadenas et al. 2009) via hypergeometric test followed by FDR correction (Benjamini and Hochberg 1995). Radar charts were used to represent the percentage of functional modules. 


\section{Identification of TFs}

The DEGs were annotated and classified as transcription factors using PlantTFact (http://plantgrn.noble.org/ PlantTFcat/) (Dai et al. 2013). All identified differentially expressed TFs were subjected to next analysis step.

\section{Construction and topology analysis of regulatory network}

Gene Set Enrichment (GSE) analysis based on Fisher exact test was applied to assign significant sub-networks among TF genes in each drought period at p-values $\leq 0.05$ using Pathway Studio software 7 (Nikitin et al. 2003; Subramanian et al. 2005). Hierarchical regulatory networks were constructed through connecting all obtained significant subnetworks using same software. Topology analysis of constructed networks was carried out by Cytoscape software 3.1.1 (Scardoni et al. 2009; Shannon et al. 2003). Betweenness and closeness centrality as well as edge betweenness parameters were considered ad major centrality parameters to find TF that has largest influence on the connection of nodes through the networks (Boccaletti et al. 2006; Girvan and Newman 2002).

\section{Conclusion}

Study of regulatory networks during different period of drought stress clearly demonstrated that plant recruits different but somewhat overlapping strategies to cope with stress in a long period of time. In each drought period, specific or common signaling pathways are activated using several numbers of transcription factors. Transcription factors related to auxin signaling namely ARR5 and ARR6 were highlighted in all drought periods indicating they are interesting candidate genes to further studies. In addition, $A B F 3$ gene which represented in D1 and D2 networks as main nod, because of its broad spectrum of target genes, has great potential to researchers for manipulate plant drought tolerance. Finally, SIGs transcription regulators may enable researchers to improve photosynthesis and yield stability under drought stress, which is the ultimate goal of plant breeders.

\section{Acknowledgements}

We thank Dr. Shamloo-Dashtpagerdi Roohollah and Dr. Talebi Majid for their support, stimulating discussions, and helpful comments. We are also grateful for the support of the biotech department of Agricultural University, Isfahan University of Technology.

\section{References}

Acharya BR \& Assmann SM (2009) Hormone interactions in stomatal function. Plant molecular biology 69(4):451-462

Ahuja I, de Vos RC, Bones AM \& Hall RD (2010) Plant molecular stress responses face climate change. Trends in plant science 15(12):664-674

Alqurashi M, Gehring C \& Marondedze C (2016) Changes in the Arabidopsis thaliana Proteome Implicate cAMP in Biotic and Abiotic Stress Responses and Changes in Energy
Metabolism. International journal of molecular sciences 17(6):852

Anjum S, Wang L, Farooq M, Khan I \& Xue L (2011) Methyl jasmonate-induced alteration in lipid peroxidation, antioxidative defence system and yield in soybean under drought. Journal of Agronomy and Crop Science 197(4):296-301

Ashraf M (2010) Inducing drought tolerance in plants: recent advances. Biotechnology advances 28(1):169-183

Benjamini $Y$ \& Hochberg $Y$ (1995) Controlling the false discovery rate: a practical and powerful approach to multiple testing. Journal of the Royal Statistical Society. Series B (Methodological):289-300

Blazejczyk M, Miron M \& Nadon R (2007) FlexArray: A statistical data analysis software for gene expression microarrays. Genome Quebec, Montreal, Canada 2007

Boccaletti S, Latora V, Moreno Y, Chavez M \& Hwang D-U (2006) Complex networks: Structure and dynamics. Physics reports 424(4):175-308

Chen M-X, Lung S-C, Du Z-Y \& Chye M-L (2014) Engineering plants to tolerate abiotic stresses. Biocatalysis and Agricultural Biotechnology 3(1):81-87

Chi W, He B, Mao J, Jiang J \& Zhang L (2015) Plastid sigma factors: their individual functions and regulation in transcription. Biochimica et Biophysica Acta (BBA)Bioenergetics 1847(9):770-778

Dai X, Sinharoy S, Udvardi M \& Zhao P (2013) PlantTFcat: an online plant transcription factor and transcriptional regulator categorization and analysis tool. BMC Bioinformatics 14(1):321

Duque AS, Farinha AP, da Silva AB, de Almeida AM, Santos D, da Silva JM, Fevereiro P \& de Sousa Araújo S (2013) Abiotic stress responses in plants: unraveling the complexity of genes and networks to survive. INTECH Open Access Publisher

Farooq M, Hussain M, Wahid A \& Siddique KHM (2012) Drought Stress in Plants: An Overview. In: Aroca R (ed) Plant Responses to Drought Stress. Springer Berlin Heidelberg, p 1-33

Farooq M, Wahid A \& Kobayashi N (2009) Plant drought stress: effects, mechanisms and management. Agron. Sustain. Dev. 29(1):185-212

Fox RJ \& Dimmic MW (2006) A two-sample Bayesian t-test for microarray data. BMC bioinformatics 7(1):126

Fujita $Y$, Fujita M, Shinozaki K \& Yamaguchi-Shinozaki $K$ (2011) ABA-mediated transcriptional regulation in response to osmotic stress in plants. Journal of plant research 124(4):509-525

Gehan MA, Greenham K, Mockler TC \& McClung CR (2015) Transcriptional networks-crops, clocks, and abiotic stress. Current opinion in plant biology 24:39-46

Gigolashvili T, Engqvist M, Yatusevich R, Müller C \& Flügge UI (2008) HAG2/MYB76 and HAG3/MYB29 exert a specific and coordinated control on the regulation of aliphatic glucosinolate biosynthesis in Arabidopsis thaliana. New Phytologist 177(3):627-642

Girvan M \& Newman ME (2002) Community structure in social and biological networks. Proceedings of the national academy of sciences 99(12):7821-7826

Golldack D, Lüking I \& Yang O (2011) Plant tolerance to drought and salinity: stress regulating transcription factors and their functional significance in the cellular transcriptional network. Plant cell reports 30(8):1383-1391 
Gul A, Ahad A, Akhtar S, Ahmad Z, Rashid B \& Husnain T (2016) Microarray: gateway to unravel the mystery of abiotic stresses in plants. Biotechnology letters 38(4):527543

Ha S, Vankova R, Yamaguchi-Shinozaki K, Shinozaki K \& Tran L-SP (2012) Cytokinins: metabolism and function in plant adaptation to environmental stresses. Trends in plant science 17(3):172-179

Harb A, Krishnan A, Ambavaram MM \& Pereira A (2010) Molecular and physiological analysis of drought stress in Arabidopsis reveals early responses leading to acclimation in plant growth. Plant Physiology 154(3):1254-1271

Irizarry RA, Hobbs B, Collin F, Beazer-Barclay YD, Antonellis KJ, Scherf U \& Speed TP (2003) Exploration, normalization, and summaries of high density oligonucleotide array probe level data. Biostatistics 4(2):249-264

Jammes F, Song C, Shin D, Munemasa S, Takeda K, Gu D, Cho D, Lee S, Giordo R \& Sritubtim S (2009) MAP kinases MPK9 and MPK12 are preferentially expressed in guard cells and positively regulate ROS-mediated $A B A$ signaling. Proceedings of the National Academy of sciences 106(48):20520-20525

Kathiresan A, Lafitte H, Chen J, Mansueto L, Bruskiewich R \& Bennett J (2006) Gene expression microarrays and their application in drought stress research. Field Crops Research 97(1):101-110

Koschützki D \& Schreiber F (2008) Centrality analysis methods for biological networks and their application to gene regulatory networks. Gene regulation and systems biology 2:193

Kunikowska A, Byczkowska A, Doniak M \& Kaźmierczak A (2013) Cytokinins résumé: their signaling and role in programmed cell death in plants. Plant cell reports 32(6):771-780

Liao $\mathrm{Y}$, Zou H-F, Wang H-W, Zhang W-K, Ma B, Zhang J-S \& Chen S-Y (2008) Soybean GmMYB76, GmMYB92, and GmMYB177 genes confer stress tolerance in transgenic Arabidopsis plants. Cell research 18(10):1047-1060

Liu Y (2012) Roles of mitogen-activated protein kinase cascades in ABA signaling. Plant cell reports 31(1):1-12

Maruyama K, Todaka D, Mizoi J, Yoshida T, Kidokoro S, Matsukura S, Takasaki H, Sakurai T, Yamamoto YY \& Yoshiwara K (2012) Identification of cis-acting promoter elements in cold-and dehydration-induced transcriptional pathways in Arabidopsis, rice, and soybean. DNA research 19(1):37-49

Mehrotra R, Bhalothia P, Bansal P, Basantani MK, Bharti V \& Mehrotra S (2014) Abscisic acid and abiotic stress tolerance-Different tiers of regulation. Journal of plant physiology 171(7):486-496

Mishra NS, Tuteja R \& Tuteja N (2006) Signaling through MAP kinase networks in plants. Archives of Biochemistry and Biophysics 452(1):55-68

Mohanty B, Kitazumi A, Cheung CM, Lakshmanan M, Benildo G, Jang I-C \& Lee D-Y (2016) Identification of candidate network hubs involved in metabolic adjustments of rice under drought stress by integrating transcriptome data and genome-scale metabolic network. Plant Science 242:224-239

Nakashima K, Ito Y \& Yamaguchi-Shinozaki K (2009) Transcriptional regulatory networks in response to abiotic stresses in Arabidopsis and grasses. Plant Physiology 149(1):88-95
Nikitin A, Egorov S, Daraselia N \& Mazo I (2003) Pathway studio-the analysis and navigation of molecular networks. Bioinformatics 19(16):2155-2157

Nogales-Cadenas R, Carmona-Saez P, Vazquez M, Vicente C, Yang X, Tirado F, Carazo JM \& Pascual-Montano A (2009) GeneCodis: interpreting gene lists through enrichment analysis and integration of diverse biological information. Nucleic acids research 37(suppl 2):W317-W322

Oh S-J, Song SI, Kim YS, Jang H-J, Kim SY, Kim M, Kim Y-K, Nahm BH \& Kim J-K (2005) Arabidopsis CBF3/DREB1A and $A B F 3$ in transgenic rice increased tolerance to abiotic stress without stunting growth. Plant Physiology 138(1):341-351

Orozco-Cárdenas ML, Narváez-Vásquez J \& Ryan CA (2001) Hydrogen peroxide acts as a second messenger for the induction of defense genes in tomato plants in response to wounding, systemin, and methyl jasmonate. The Plant Cell 13(1):179-191

Pérez-Salamó I, Papdi C, Rigó G, Zsigmond L, Vilela B, Lumbreras V, Nagy I, Horváth B, Domoki M \& Darula Z (2014) The Heat Shock Factor A4A Confers Salt Tolerance and Is Regulated by Oxidative Stress and the MitogenActivated Protein Kinases MPK3 and MPK6. Plant physiology 165(1):319-334

Pessarakli M (2011) Handbook of plant and crop stress, edited by Mohammad Pessarakli. Books in soils, plants, and the environment.

Quan $\amalg$, Zhang B, Shi WW \& Li HY (2008) Hydrogen peroxide in plants: a versatile molecule of the reactive oxygen species network. Journal of Integrative Plant Biology 50(1):2-18

Raghavendra AS, Gonugunta VK, Christmann A \& Grill E (2010) ABA perception and signalling. Trends in plant science 15(7):395-401

Rasheed S, Bashir K, Matsui A, Tanaka M \& Seki M (2016) Transcriptomic Analysis of Soil-Grown Arabidopsis thaliana Roots and Shoots in Response to a Drought Stress. Frontiers in plant science 7

Scardoni G, Petterlini M \& Laudanna C (2009) Analyzing biological network parameters with CentiScaPe. Bioinformatics 25(21):2857-2859

Seki M, Kamei A, Yamaguchi-Shinozaki K \& Shinozaki K (2003) Molecular responses to drought, salinity and frost: common and different paths for plant protection. Current Opinion in Biotechnology 14(2):194-199 doi:http://dx.doi.org/10.1016/S0958-1669(03)00030-2

Shamloo-Dashtpagerdi R, Razi H \& Ebrahimie E (2015) Mining expressed sequence tags of rapeseed (Brassica napus L.) to predict the drought responsive regulatory network. Physiology and Molecular Biology of Plants 21(3):329-340

Shannon P, Markiel A, Ozier O, Baliga NS, Wang JT, Ramage D, Amin N, Schwikowski B \& Ideker T (2003) Cytoscape: a software environment for integrated models of biomolecular interaction networks. Genome research 13(11):2498-2504

Subramanian A, Tamayo P, Mootha VK, Mukherjee S, Ebert BL, Gillette MA, Paulovich A, Pomeroy SL, Golub TR \& Lander ES (2005) Gene set enrichment analysis: a knowledge-based approach for interpreting genome-wide expression profiles. Proceedings of the National Academy of Sciences of the United States of America 102(43):1554515550 
Tabas-Madrid D, Nogales-Cadenas R \& Pascual-Montano A (2012) GeneCodis3: a non-redundant and modular enrichment analysis tool for functional genomics. Nucleic acids research 40(W1):W478-W483

Tuberosa R \& Salvi S (2006) Genomics-based approaches to improve drought tolerance of crops. Trends in plant science 11(8):405-412

Walia $\mathrm{H}$, Wilson $\mathrm{C}$, Condamine $\mathrm{P}$, Liu $\mathrm{X}$, Ismail AM \& Close TJ (2007) Large-scale expression profiling and physiological characterization of jasmonic acid-mediated adaptation of barley to salinity stress. Plant, Cell \& Environment 30(4):410-421

Wang Y, Li L, Ye T, Zhao S, Liu Z, Feng YQ \& Wu Y (2011) Cytokinin antagonizes ABA suppression to seed germination of Arabidopsis by downregulating $A B I 5$ expression. The Plant Journal 68(2):249-261

Wong C, Li Y, Whitty B, Diaz-Camino C, Akhter S, Brandle J, Golding G, Weretilnyk E, Moffatt B \& Griffith M (2005) Expressed sequence tags from the Yukon ecotype of Thellungiella reveal that gene expression in response to cold, drought and salinity shows little overlap. Plant molecular biology 58(4):561-574

Xing $Y$, Jia $W$ \& Zhang $J$ (2008) AtMKK1 mediates ABA-induced CAT1 expression and $\mathrm{H} 2 \mathrm{O} 2$ production via AtMPK6-coupled signaling in Arabidopsis. The Plant Journal 54(3):440-451

Xu Z, Jiang Y \& Zhou G (2015) Response and adaptation of photosynthesis, respiration, and antioxidant systems to elevated $\mathrm{CO} 2$ with environmental stress in plants. Frontiers in plant science 6
Yamaguchi-Shinozaki K \& Shinozaki K (2005) Organization of $<\mathrm{i}>\mathrm{cis}</ \mathrm{i}>$-acting regulatory elements in osmotic-and cold-stress-responsive promoters. Trends in plant science 10(2):88-94

Yamaguchi-Shinozaki K \& Shinozaki K (2006) Transcriptional regulatory networks in cellular responses and tolerance to dehydration and cold stresses. Annu. Rev. Plant Biol. 57:781-803

Yamori W (2016) Photosynthetic response to fluctuating environments and photoprotective strategies under abiotic stress. Journal of plant research 129(3):379-395

Yoshida T, Fujita Y, Sayama H, Kidokoro S, Maruyama K, Mizoi J, Shinozaki K \& Yamaguchi-Shinozaki K (2010) AREB1, AREB2, and ABF3 are master transcription factors that cooperatively regulate $A B R E-$ dependent $A B A$ signaling involved in drought stress tolerance and require $A B A$ for full activation. The Plant Journal 61(4):672-685

Zeller G, Henz SR, Widmer CK, Sachsenberg T, Rätsch G, Weigel D \& Laubinger S (2009) Stress-induced changes in the Arabidopsis thaliana transcriptome analyzed using whole-genome tiling arrays. The Plant Journal 58(6):10681082

Zhang X, Zhang L, Dong F, Gao J, Galbraith DW \& Song C-P (2001) Hydrogen peroxide is involved in abscisic acidinduced stomatal closure in Vicia faba. Plant physiology 126(4):1438-1448 